\title{
EMSY-Like Genes Are Required for Full RPP7-Mediated Race-Specific Immunity and Basal Defense in Arabidopsis
}

\author{
Tokuji Tsuchiya and Thomas Eulgem \\ Center for Plant Cell Biology, Institute for Integrative Genome Biology, Department of Botany and Plant Sciences, University \\ of California at Riverside, CA 92521, U.S.A.
}

Submitted 16 May 2011. Accepted 3 August 2011.

\begin{abstract}
The Arabidopsis thaliana gene enhanced downy mildew 2 (EDM2) encodes a nuclear protein required for $R P P 7-m e-$ diated race-specific disease resistance against Hyaloperonospora arabidopsidis, proper floral transition and additional developmental processes. Transcript levels of the diseaseresistance gene $R P P 7$ are enhanced by EDM2 while those of the floral suppressor $F L C$ are repressed by EDM2. By yeast two-hybrid screening for EDM2-interacting proteins, we identified AtEML1, a member of a small group of four Arabidopsis proteins containing an EMSY N-terminal domain, a central Agenet domain, and a C-terminal coiledcoil motif. Using T-DNA mutants combined with silencing by artificial microRNAs, we found $A t E M L 1, A t E M L 2$, and, likely, $A t E M L 4$ to contribute to RPP7-mediated immunity. Besides this, AtEML1 and AtEML2 participate in a second EDM2-dependent function and affect floral transition. Unlike $E D M 2$, whose role in immunity appears to be limited to $R P P 7-$ mediated disease resistance, some AtEML members contribute to basal defense, an unspecific general defense mechanism. Domain architectures of EDM2 as well as AtEML proteins suggest roles of these proteins in the regulation of chromatin states. Thus, possible cooperation of AtEML members with EDM2 at the level of chromatin dynamics may link race-specific pathogen recognition to general defense mechanisms.
\end{abstract}

Two interconnected regulatory systems are known to mediate nonself recognition and innate immunity in plants (Dodds and Rathjen 2010). Pattern-triggered immunity (PTI) is based on specific recognition of broadly conserved molecular signatures of pathogens termed pathogen- or microbe-associated molecular patterns (PAMPs or MAMPs) by plant pattern-recognition receptors (PRR) (Segonzac and Zipfel 2011). Effector molecules secreted by virulent pathogens can attenuate PTI, leading to a weakened immune response termed basal defense (Nimchuk et al. 2003). Recognition of effectors by plant disease resistance $(\mathrm{R})$ proteins results in strong effector-triggered immunity (ETI), which efficiently prevents infections by avirulent biotrophic pathogens (Chisholm et al. 2006; Jones and Dangl 2006). ETI is race specific, because effectors and their complementary cognate $\mathrm{R}$ proteins are typically only present in pairs of corresponding pathogen/plant races and are not con-

Corresponding author: T. Eulgem; E-mail: thomas.eulgem@ucr.edu

* The $e$-Xtra logo stands for "electronic extra" and indicates that four supplementary figures and one supplementary table are published online. served at the species level. A hallmark of ETI is the hypersensitive response (HR), the programmed death of plant cells at pathogen infection sites.

A complex regulatory network integrates distinct defense signals and coordinates various types of immune responses (Sato et al. 2010; Tsuda et al. 2009). Although both PTI and ETI involve a common set of downstream responses, such as transcriptional reprogramming of a core set of defense genes (Eulgem et al. 2004), production of reactive oxygen intermediates (ROI) (Torres et al. 2002), and the accumulation of salicylic acid (SA) and jasmonic acid (Glazebrook 2005), regulatory circuits connecting PAMP or MAMP and effector recognition are still largely unknown.

Several recent studies uncovered connections between floral transition pathways and innate immunity (Jin et al. 2008; March-Diaz et al. 2008; Tör et al. 2002; Tsuchiya and Eulgem 2010a; Wang et al. 2011). We reported that enhanced downy mildew 2 (EDM2), which is required for race-specific resistance of Arabidopsis against the Hiks1 isolate of the oomycete Hyaloperonospora arabidopsidis, also positively affects floral transition (Tsuchiya and Eulgem 2010a). Like mutants of the autonomous floral promotion pathway, mutants of EDM2 show a photoperiod-independent late-flowering phenotype. Further analysis revealed that EDM2 has a promoting affect on floral transition by suppressing transcript levels of the floral repressor gene $F L C$. EDM2 appears to physically interact with the protein kinase WNK8, which acts in this pathway upstream from EDM2 and counteracts EDM2 function.

EDM2 is a nuclear protein containing an acidic N-terminal region, two PHD-finger-like domains, a G $\gamma$-like (GGL)-related protein-protein interaction domain we termed the plant GGLrelated (PGR) domain, two putative bipartite nuclear localization signals, the ELP domain, which is a conserved sequence of unknown function, and a C-terminal proline-rich region (Eulgem et al. 2007; Tsuchiya and Eulgem 2010a). Physical interactions with WNK8 involve the PGR domain of EDM2 and a region of the kinase containing a putative coiled-coil structure (Tsuchiya and Eulgem 2010a). Although WNK8 affects the roles of EDM2 in flowering time control and other developmental processes (Tsuchiya and Eulgem 2010b), it seems not to have any detectable effect on EDM2-mediated immunity (Tsuchiya and Eulgem 2010a).

EDM2 enhances transcript levels of the $R$ gene $R P P 7$, which is required for race-specific immunity of Arabidopsis against H. arabidopsidis Hiks1 (Eulgem et al. 2007). The role of EDM2 in immunity is highly specific. Whereas many other regulators required for $R$ gene function have broader roles in immunity, EDM2 appears only to affect disease resistance against $H$. 
arabidopsidis Hiks1. It does not contribute to basal defense and no $R$ genes, besides $R P P 7$, have been reported to be dependent on EDM2. Mutations in EDM2 phenocopy defenserelated effects of rpp7 mutants (Eulgem et al. 2007). In both types of mutants, $H$. arabidopsidis Hiks 1 -induced HR and ROI production are abolished and expression of a common set of defense-related genes is affected. Taken together, these observations suggested that EDM2 acts at least in part through $R P P 7$, by regulating transcript levels of this $R$ gene.

Here, we report data functionally linking EDM2 to members of an Arabidopsis family of four EMSY-like (AtEML) proteins. AtEML proteins have possible roles in chromatin regulation and are related to the BRCA2-interacting human oncoprotein EMSY (Hughes-Davies et al. 2003). AtEML1 and AtEML2, along with at least one additional member of this set of genes, have overlapping roles in activating EDM2- or RPP7-dependent immunity against $H$. arabidopsidis Hiks1, while AtEML1 and $A t E M L 2$ appear to counteract the promoting effect of $E D M 2$ on floral transition. In contrast to edm2 mutants, knockdown of $A t E M L$ members reduced basal defense against a virulent $H$. arabidopsidis isolate. Thus, AtEML proteins have a broader role in plant immunity than EDM2. By interacting with EMSY-like proteins, EDM2 may bridge between an $H$. arabidopsidis Hiks1-specific recognition mechanism and a more general defense function contributing to basal defense.

\section{RESULTS}

EDM2 can physically interact with AtEML1 in plant nuclei.

To identify EDM2-interacting proteins, we performed a yeast two-hybrid screen using the central portion of EDM2 (EDM2 214-1204) fused to the GAL4 DNA-binding domain as a bait and several activation domain expression libraries representing pooled cDNAs from untreated and $H$. arabidopsidisinfected Col-0 seedlings (Tsuchiya and Eulgem 2010a). Two independent, positive clones we identified encode the same protein containing an EMSY N-terminal (ENT) domain, a central Agenet domain, and a putative C-terminal coiled-coil structure. We termed this protein AtEMSY-like 1 (AtEML1; AT3G12140). The ENT domain of AtEML1 strongly resembles that of the human oncoprotein EMSY (Hughes-Davies et al. 2003). The Arabidopsis genome harbors three additional genes encoding proteins that are structurally closely related to AtEML1 and contain an N-terminal ENT domain, a central Agenet domain, and a C-terminal coiled-coil motif (Supplementary Fig. S1). We termed them AtEML2 (AT5G06780), AtEML3 (AT5G13020), and AtEML4 (AT2G44440). We collectively refer to this set of four genes as AtEML1/2/3/4.

Our previous microarray analyses (Tsuchiya and Eulgem 2010b) revealed that constitutive transcript levels of all four $A t E M L$ genes are low (close to the noise range), with AtEML4 transcript levels being the lowest. Relative to those of AtEML4, constitutive transcript levels of AtEML1, AtEML2, or AtEML3 appear to be elevated by 2.6-, 3.7-, or 2.8-fold, respectively. Examining publicly accessible microarray data, we found transcripts of none of the AtEML1/2/3/4 members to exhibit clear inducibility by any of a variety of biotic or abiotic stresses or hormone treatments. The only exception seems to be a moderate upregulation of AtEML2 transcripts by heat stress.

Three splice forms are reported for AtEML1. AtEML1 version 1 (v1) and version 2 (v2) encode identical proteins. In the protein encoded by the third splice version (v3), the last 15 amino acids are replaced by a $\mathrm{C}$-terminal extension comprised of 74 amino acids. By transiently expressing binary CaMV35S:GFP-AtEML1 expression constructs in Nicotiana benthamiana leaves, we found both AtEML1-v1 and AtEML1-v3 to localize to the nucleus, while green fluorescent protein (GFP) alone also could be detected in the cell periphery (Fig. 1A).

To examine whether EDM2 and AtEML1 can physically interact in planta, we performed bimolecular fluorescence complementation (BiFC) assays (Citovsky et al. 2006) in tobacco leaves (Fig. 1B). Transient co-expression of the N-terminal half of yellow fluorescent protein (YFP) fused to the $\mathrm{N}$ terminus of the full-length EDM2 protein (nYFP-EDM2) and the Cterminal half of YFP fragment fused to full-length AtEML1-v1 (cYFP-AtEML1-v1) resulted in strong nuclear YFP fluorescence, indicating physical in vivo interactions between EDM2 and AtEML1 in nuclei. We obtained similar results with the closest AtEML1 paralog, AtEML2. No interactions were observed between nYFP-EDM2 and c-YFP-AtEML1-v3 or isolated cYFP (Fig. 1B).

\section{AtEML1 and its Arabidopsis paralogs have overlapping roles in EDM2-mediated immunity.}

Using a set of T-DNA mutants and transgenic Arabidopsis lines expressing artificial microRNAs (amiRNAs), we tested if AtEML1/2/3/4 contribute to the immune function of EDM2. Because EDM2 and RPP7 specifically mediate immunity of the Arabidopsis Col-0 and Col-5 accessions to H. arabidopsidis Hiks1, all lines used in this study are of the Col-0 background (Table 1). We tested three independent mutants for AtEML1 (eml1-1, eml1-2, and eml1-3), one AtEML2 mutant (eml2-1), one AtEML3 mutant (eml3-1), an AtEML1/AtEML2 double mutant (eml1-2/eml2-1), and three independent lines expressing amiRNA co-silencing AtEML3 and AtEML4 transcripts in the emll-2/eml2-1 background (eml-quadl, emlquad2, and eml-quad3). None of the tested single or double eml mutants or the eml-quad lines showed any obvious growth-related phenotype or developmental defects.

The intronic insertions of emll-1, emll-2, and eml2-1 caused only a mild reduction of the transcript levels of AtEML1 or AtEML2, respectively (Supplementary Fig. S2A and B). AtEML1 transcripts are expressed at wild-type levels in the emll-3 mutant, which harbors an insertion in an exon of AtEML1. AtEML3 transcript levels are strongly reduced (but still detectable) in eml3-1 plants, which also contain an exonic insertion. Based on the nature of their insertion, only the emll3 and eml3-1 mutants are likely to constitute strong alleles, because the reading frames of the respective AtEML coding sequences are disrupted. The function of $A t E M L$ genes in emll-1, emll-2, or eml2-1, which contain intronic insertions, is likely to be only weakly compromised, because these mutants allow for near-wild-type levels of the respective transcripts. Only four lines of transgenic plants expressing the amiRNA cosilencing AtEML3 and AtEML4 in the eml1-2/eml2-1 background could be recovered. Three of these eml-quad lines (eml-quad1, eml-quad2, and eml-quad3) contain single transgene insertions and were further characterized. In these lines, AtEML3 and AtEM4 transcript levels were reduced by only 30 to $60 \%$ compared with wild-type levels. The low number of transgenic lines we were able to recover and the partial nature of AtEML3 and AtEML4 silencing observed in them may indicate that full silencing of these genes is lethal.

In order to examine whether $A t E M L 1 / 2 / 3 / 4$ contribute to the defense function of $E D M 2$, we scored our set of $\mathrm{eml}$ mutants and amiRNA lines 7 days postinfection with $H$. arabidopsidis Hiks1. Individual cotyledons of the infected plants were assigned to defined "bins" based on the strongest type of infection symptom they exhibited using the following classification scale: discrete HR sites $(\mathrm{HR})<$ extended HR sites $(\mathrm{eHR})<$ trailing necrosis $(\mathrm{TN})<$ free $H$. arabidopsidis hyphae $(\mathrm{FH})<$ $H$. arabidopsidis oospores $(\mathrm{Oo})<H$. arabidopsidis sporangiophores (Sp) (Supplementary Fig. S3). The category "HR" rep- 


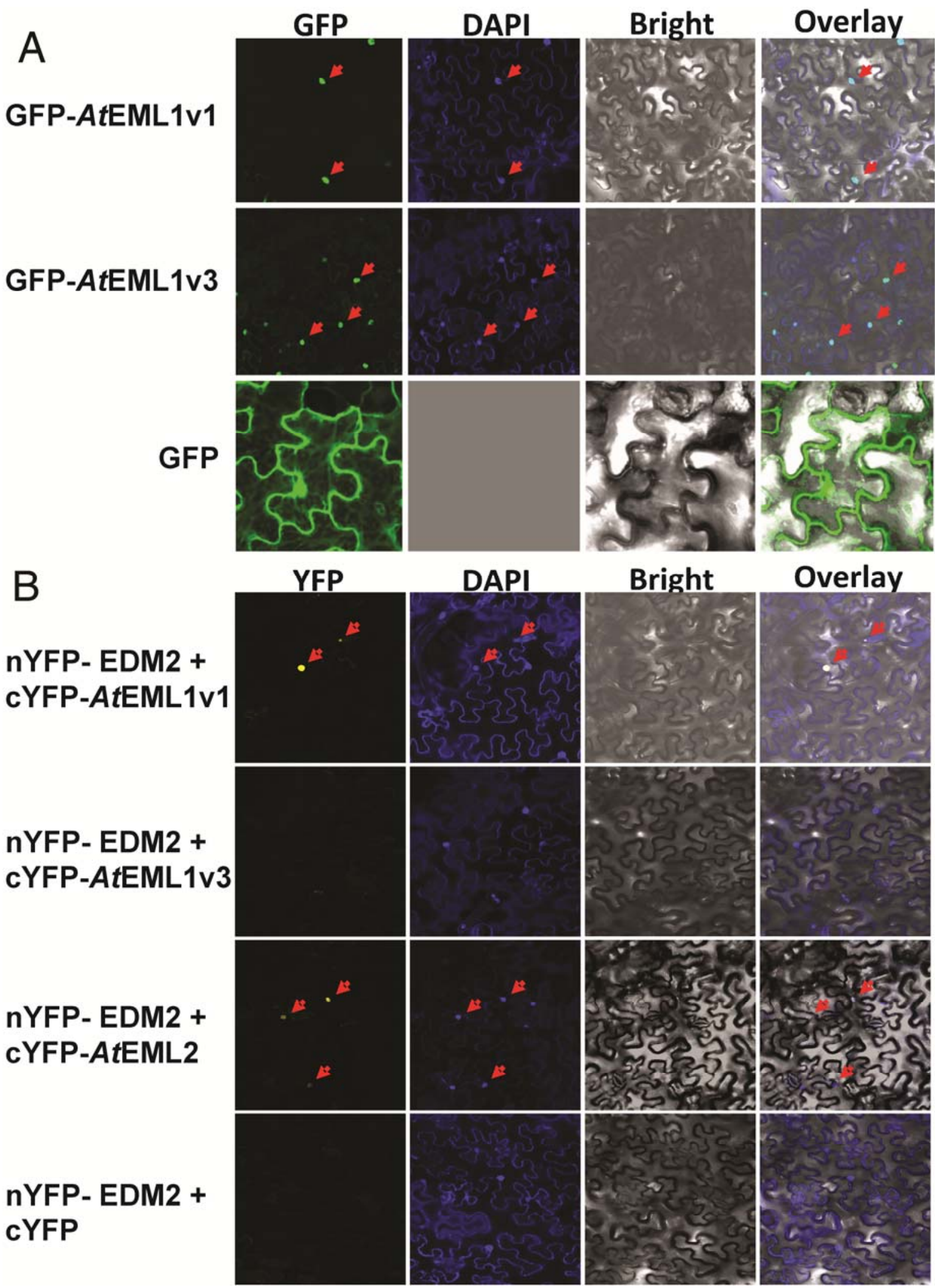

Fig. 1. Enhanced downy mildew 2 (EDM2) and EMSY-like 1 (AtEML1) are co-localized and interact under in vivo conditions in Nicotiana benthamiana nuclei. A, Subcellular localization of green fluorescent protein (GFP) fused to two different AtEML1 splice forms, GFP-AtEML1 v1 and GFP-AtEML1 v3, in transiently transformed $N$. benthamiana leaf tissue. Fluorescence was observed by confocal microscopy. B, Bimolecular fluorescence complementation (BiFC) analysis of EDM2/AtEML1 and EDM2/AtEML2 interactions in vivo. BiFC constructs were transformed into N. benthamiana leaf epidermal cells by agroinfiltration. Four days postinoculation, yellow fluorescent protein (YFP) fluorescence was observed by confocal microscopy. YFP fluorescence results from complementation between the N-terminal (nYFP) and C-terminal (cYFP) parts of YFP fused to the N-termini of the respective tested proteins. Nuclear interactions with nYFP-EDM2 were only observed for cYFP-AtELM1-v1 and cYFP-AtEML2 but not cYFP-AtELM1-v3 or cYFP alone. A and B, Representative examples. 4',6-Diamidino-2-phenylindole (DAPI) staining was performed to specify locations of nuclei. Overlay: merged images of GFP or YFP, DAPI, and bright field. B, In any photographed tissue section, only a small number of fluorescing nuclei were in the plane of focus. However, we reproducibly observed for co-expression of nYFP-EDM2 with cYFP-AtEML1v1 or cYFP-AtEML2 a large number of BiFC-positive nuclei. 
resents a strong immune response not permitting the development of $H$. arabidopsidis hyphae, while "eHR" indicates that immunity is partially weakened, allowing limited growth of hyphae, which are still surrounded by plant cells undergoing HR cell death. In the case of trailing necrosis, HR trails extended $H$. arabidopsidis hyphae. Free hyphae are not surrounded or trailed by HR. Occasionally, the formation of sexual oospores is observed during successful $H$. arabidopsidis infections (Eulgem et al. 2007). Oospores as well as sporangiophores, which serve the release of asexual spores, represent the successful conclusion of the $H$. arabidopsidis infection cycle (Holub and Beynon 1996).

None of the tested $\mathrm{eml}$ lines showed as strong a reduction of $H$. arabidopsidis Hiks1 resistance as edm2-2 (Eulgem et al. 2007), which has nearly completely lost its immunity to this pathogen (Fig. 2A) (Tsuchiya and Eulgem 2010a). In Col-0 plants, there were no free $H$. arabidopsidis Hiks1 hyphae, oospores, or sporangiophores. We occasionally observed free hyphae in emll single mutants. However, only the eml1-2/eml2-1 double mutant and the eml-quad lines showed a significant in-

Table 1. Mutant and transgenic Arabidopsis lines used in this study

\begin{tabular}{lll}
\hline Name of line & \multicolumn{1}{c}{ AGI number for affected genes ${ }^{\text {a }}$} & \\
\hline emsy-like 1-1 (eml1-1) & AT3G12140 & Comments \\
emsy-like 1-2 (eml1-2) & AT3G12140 & T-DNA insertion in intron of 5' untranslated region (UTR) (SALK_108872) \\
emsy-like 1-3 (eml1-3) & AT3G12140 & T-DNA insertion in fifth intron (SALK_114038) \\
emsy-like 2-1 (eml2-1) & AT5G06780 & T-DNA insertion on sixth exon (SALK_077088) \\
emsy-like 3-1 (eml3-1) & AT5G13020 & T-DNA insertion in intron of 5' UTR (SALK_116222) \\
eml1-2/eml2-1 & AT3G12140, AT5G06780 & T-DNA insertion in second exon (SALK_106147) \\
eml-quad-1 & AT3G12140, AT5G06780, AT5G13020, & SALK_114038/ SALK_116222 double mutant \\
& AT2G44440 & eml1-2/eml2-1 background \\
eml-quad-2 & Same as eml-quad-1 & Second independent eml-quad transgenic line \\
eml-quad-3 & Same as eml-quad-1 & Third independent eml-quad transgenic line \\
\hline
\end{tabular}

${ }^{\mathrm{a}} \mathrm{AGI}=$ Arabidopsis Genome Initiative.
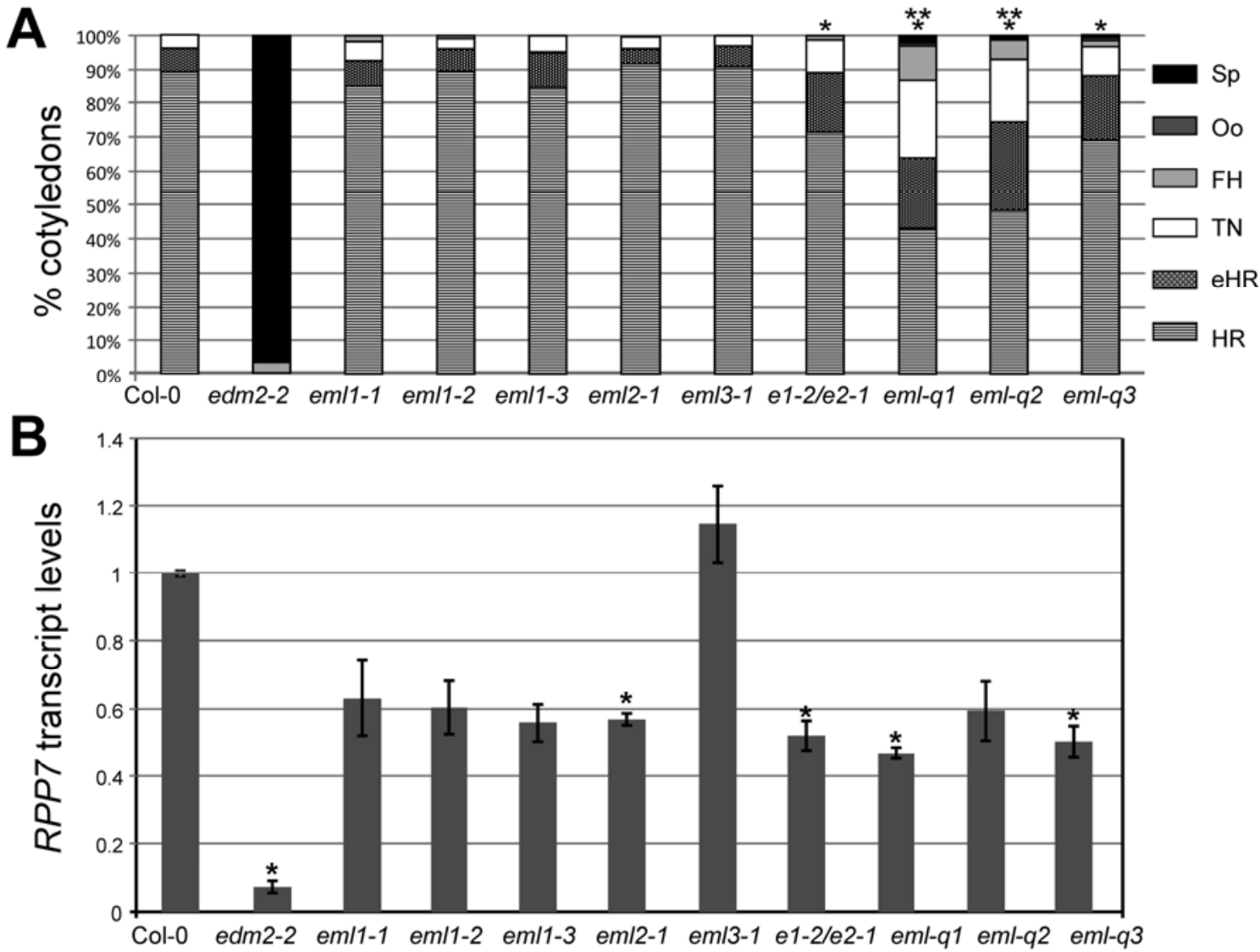

Fig. 2. Knock-down of EMSY-like (AtEML) genes affects enhanced downy mildew 2 (EDM2)-dependent immunity. A, Two-week-old Arabidopsis seedlings were spray infected with Hyaloperonospora arabidopsidis Hiks 1 spores at $5 \times 10^{4} / \mathrm{ml}$ and scored for disease symptoms 7 days later. Individual cotyledons of the infected plants were assigned to defined categories based on the strongest type of infection symptom they exhibited using the following classification scale: discrete hypersensitive response (HR) sites < extended HR sites (eHR) < trailing necrosis $(\mathrm{TN})<$ free H. arabidopsidis hyphae $(\mathrm{FH})<H$. arabidopsidis oospores $(\mathrm{Oo})<H$. arabidopsidis sporangiophores (Sp) (see also Supplementary Figure S3). Based on the frequencies of eHR- and TN-containing cotyledons (*), $\chi^{2}$ tests (Hartl and Jones 1998$)$ showed highly significant differences $(P<0.01)$ between Col-0 on the one hand and eml1-2/eml2-1 (e1-1/e2-1) as well as all three eml-quad (eml-q) lines on the other hand. Similarly, frequencies of eHR-, TN-, and FH-containing cotyledons differed highly significantly $(P<0.01)$ in eml-quadl and eml-quad2 from those in the eml1-2/eml2-1 line (**). B, RPP7 transcript levels in 2-week-old seedlings of the indicated Arabidopsis lines determined by quantitative real-time reverse-transcription polymerase chain reaction. Data shown are based on averages from two biological replicates, each representing three technical replicates. Error bars represent standard deviations; ${ }^{*}$ symbols mark significant changes $(P<0.05)$ relative to Col-0 as determined by Student's $t$ tests, which were performed as described previously (Yuan et al. 2006). 
crease of infection symptoms. The eml1-2/eml2-1 double mutant exhibited a more than twofold higher frequency of eHR and $\mathrm{TN}$ cotyledons than Col-0. Because a significant reduction of $H$. arabidopsidis Hiks1 resistance was observed in the eml1-2/eml2-1 double mutant but not in the eml1-2 or eml2-1 single mutants, both AtEML1 and AtEML2 play redundant roles in this defense mechanism.

Compared with eml1-2/eml2-1 plants, the reduction of immunity was more pronounced in the eml-quad lines, which not only showed a clear increase in the number of eHR and TN cotyledons but also exhibited more cotyledons with free hyphae. Based on the occurrence of eHR-, TN-, and FH-containing cotyledons, the eml-quadl and eml-quad2 lines were significantly more susceptible than the emll-2/eml2-1 double mutant. In addition, these two amiRNA lines occasionally allowed for the development of oospores and sporangiophores. Thus, either both AtEML3 and AtEML4 together or one of them alone enhances the immune function of AtEML1 and AtEML2. Taken together, our results show that AtEML1 and AtEML2 along with one or both additional members of the AtEML1/2/3/4 set act redundantly in promoting immunity against $H$. arabidopsidis Hiks1, which is also dependent on EDM2.

EDM2 is likely to contribute to $H$. arabidopsidis Hiks1 resistance by enhancing transcript levels of the $R$ gene $R P P 7$ (Eulgem et al. 2007; Tsuchiya and Eulgem 2010a). Quantitative real-time reverse-transcription polymerase chain reaction (qPCR) showed RPP7 transcript levels to be reduced by 40 to $50 \%$ in all tested eml and eml-quad lines, except for eml3-1. However, these changes were only significant for eml1-2, eml12/eml2-1, and two of the eml-quad lines (Fig. 2B). Consistent with the weaker nature of their $H$. arabidopsidis Hiks1 susceptibility phenotype, the reduction of $R P P 7$ transcript levels is less pronounced in the eml1-2/eml2-1 mutant and eml-quad lines compared with edm2-2 plants. Unlike all other tested eml mutants, eml3-1 plants clearly did not show a reduction of $R P P 7$ transcript levels. Thus, increased H. arabidopsidis Hiks1 susceptibility in the eml-quadl and eml-quad2 lines compared with the eml1-2/eml2-1 double mutant may reflect a role of AtEMLA but not AtEML3 in this EDM2-dependent defense pathway.

Although the eml2-1 single mutant also exhibited a significant reduction of $R P P 7$ transcript levels, it appears not to be compromised in $H$. arabidopsidis Hiks 1 resistance. This may indicate that AtEML genes may partially utilize defense mechanisms that are independent from RPP7 and do not correlate with the levels of suppression of this $R$ gene. The role of AtEML genes in basal defense described below supports such a scenario.

AtEML genes have a broader role in immunity than $E D M 2$.

The contribution of EDM2 to immunity seems to be limited to the $R P P 7$ pathway, which specifically mediates strong resistance to $H$. arabidopsidis Hiks1. Neither basal defense to $H$. arabidopsidis nor race-specific resistance mediated by other characterized Col-0 $R$ genes appears to be reduced in edm2 mutants (Eulgem et al. 2007; Tsuchiya and Eulgem 2010a). To test whether the defense function of $A t E M L$ genes is also limited to resistance against $H$. arabidopsidis Hiks1, we infected eml mutants and eml-quad lines with the $H$. arabidopsidis isolate Noco2, which is virulent on Col-0 plants. As expected, the edm2-2 mutant exhibited fully intact basal defense and behaved as susceptible to this $H$. arabidopsidis isolate as Col-0 plants (Fig. 3). Wild-type plants of the Arabidopsis Landsberg erecta (Ler) accession, which recognize $H$. arabidopsidis Noco 2 by the RPP5 $R$ gene (Parker et al. 1993), were fully resistant. To our surprise, all three eml-quad lines exhibited significantly increased susceptibility to $H$. arabidopsidis Noco2 allowing for a two- to threefold or higher number of $\mathrm{H}$. arabidopsidis spores to be produced than Col-0. However, compared with the massively enhanced susceptibility of the transgenic $n a h G$ line, which is known to be strongly compromised

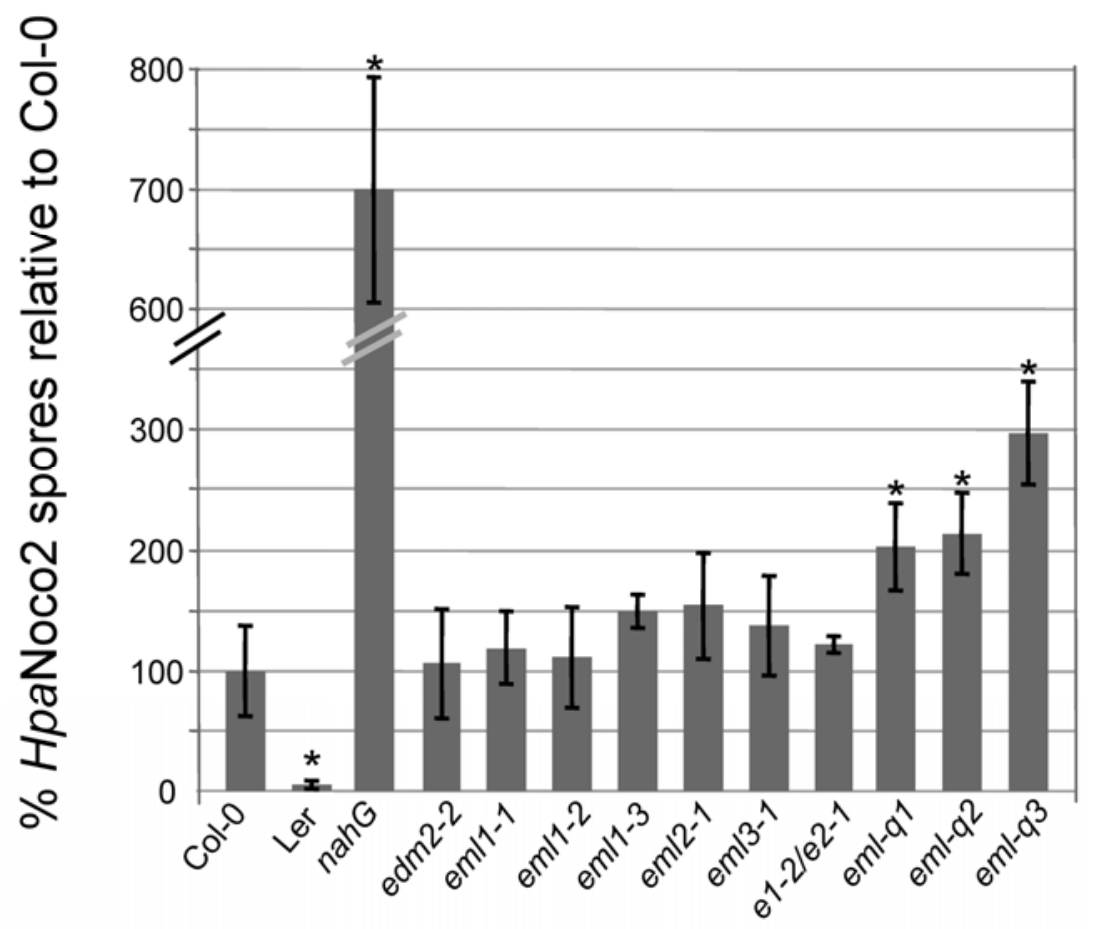

Fig. 3. EMSY-like (eml) mutants and eml-quad lines are compromised in basal defense against Hyaloperonospora arabidopsidis Noco2. Three-week-old Arabidopsis seedlings of the indicated genotypes were spray-inoculated with the virulent $H$. arabidopsidis isolate Noco 2 at $2 \times 10^{4}$ spores/ml. Numbers of spores per milligram fresh weight were counted at 7 days postinfection. Spore numbers are shown relative to those observed in Col-0 plants. Error bars represent standard deviations based on three independent biological replicates. Significant $(*, P<0.05)$ differences between average spore numbers were detected using Student's $t$ tests. 
in basal defense (Delaney et al. 1994), the reduction of basal defense in the eml-quad lines is moderate. Although our data do not allow to infer which AtEML1/2/3/4 members contribute to basal defense against $H$. arabidopsidis Noco2, clearly at least one representative of this set has a broader role in immunity than EDM2. The reduction of basal defense that we observed in eml-quad lines may reflect overlapping contributions of multiple AtEML1/2/3/4 members to this immune response, or a basal defense-related role of either AtEML3 or AtEML4.

\section{AtEML genes negatively affect floral transition.}

We previously found EDM2 to have a promoting effect on floral transition (Tsuchiya and Eulgem 2010a). To test whether AtEML1/2/3/4 also affect flowering time, we grew eml mutants and silencing lines under long-day conditions and counted the number of rosette leaves formed until bolting (Table 2). As observed previously, the edm2-2 mutant flowered late compared with Col-0. The eml1-3 mutant showed a moderate but significant early flowering phenotype, forming, on average, two to three fewer rosette leaves before bolting than Col- 0 plants. The eml2-1 mutant exhibited a similar trend, which is also significant but weaker. Floral transition was clearly accelerated in the emll-2/eml2-1 double mutant by three leaves compared with Col-0. This phenotype exceeded the weak effect observed in eml2-1 plants. The flowering phenotype of emll-2/eml2-1 plants was not further enhanced in any of the eml-quad lines.

Table 2. AtEML1 and AtEML2 negatively affect floral transition ${ }^{\mathrm{a}}$

\begin{tabular}{lcc}
\hline Line & No. of rosette leaves & $\boldsymbol{t}^{\mathbf{t}}$ test $\boldsymbol{P}$ values (versus Col-0) \\
\hline Col-0 & $12.5 \pm 0.2$ & $\ldots$ \\
edm2-2 & $17 \pm 0.5$ & $2.662 \mathrm{E}-08$ \\
emll-1 & $12.6 \pm 0.2$ & 0.749 \\
eml1-2 & $12.5 \pm 0.3$ & 0.947 \\
eml1-3 & $9.7 \pm 0.3$ & $4.175 \mathrm{E}-07$ \\
eml2-1 & $11.4 \pm 0.2$ & 0.009 \\
eml1-2/eml2-1 & $9.6 \pm 0.2$ & $4.654 \mathrm{E}-09$ \\
eml-quad-1 & $10.1 \pm 0.3$ & $1.806 \mathrm{E}-06$ \\
eml-quad-2 & $9.7 \pm 0.2$ & $2.269 \mathrm{E}-09$ \\
eml-quad-3 & $9.6 \pm 0.3$ & $3.102 \mathrm{E}-08$ \\
\hline
\end{tabular}

${ }^{\text {a }}$ Flowering time during long-day conditions.

b:Number of rosette leaves formed until bolting. Total leaves: $n=20$.
$E D M 2$ promotes floral transition by suppressing transcript levels of the floral suppressor gene FLC (Tsuchiya and Eulgem 2010a). FLC transcript levels are enhanced in edm2 mutants. Consistent with their early-flowering phenotype, we found, by qPCR, FLC transcript levels of eml1-3, eml2-1, eml1-2/eml2-1, and eml-quad plants to be significantly reduced compared with Col- 0 and to accumulate to only approximately $50 \%$ of wildtype levels (Fig. 4). Although FLC transcript levels appear also to be reduced in elm1-1 and elm1-2 plants, these transcript changes were not significant. Our data indicate that AtEML1 and $A t E M L 2$ negatively affect floral transition and counteract the promoting effect of $E D M 2$ on this developmental process. Similar to EDM2, the effect of AtEML1 and AtEML2 on floral transition is likely mediated by modulation of $F L C$ transcript levels. These findings provide further support for a functional connection between AtEML genes and EDM2.

\section{DISCUSSION}

The Arabidopsis genome harbors nine genes encoding proteins with ENT domains (Hughes-Davies et al. 2003). We limited our study to a set of four closely related members of this family, which we termed AtEML1/2/3/4, and which encode proteins containing, besides the ENT domain, a central Agenet domain and a C-terminal coiled-coil motif. We found members of this gene set to affect plant innate immunity as well as floral transition. Compared with the strong defense-related phenotypes of the edm2-2 or $n a h G$ lines that served as controls in this study, the reduction of $H$. arabidopsidis Hiks1 resistance or basal defense in eml mutant and silencing lines was only weak to moderate. Most likely, functional redundancy between individual members of the AtEML1/2/3/4 gene set as well as the use of weak alleles did not allow for a stronger expression of these phenotypes. For example, in the eml-quad lines, weak T-DNA alleles of AtEML1 and AtEML2 are combined with an amiRNA construct that only partially silences AtEML3 and AtEML4 transcripts. Nonetheless, the defense-related phenotypes we observed were very clear and reflect important contributions of the tested genes to the respective defense pathways.

AtEML1/2/3/4 have not been functionally characterized in Arabidopsis. The only plant gene of this type for which a functional study has been reported is RIFl. It encodes a nuclear protein of maize that interacts with the basic helix-loop-helix

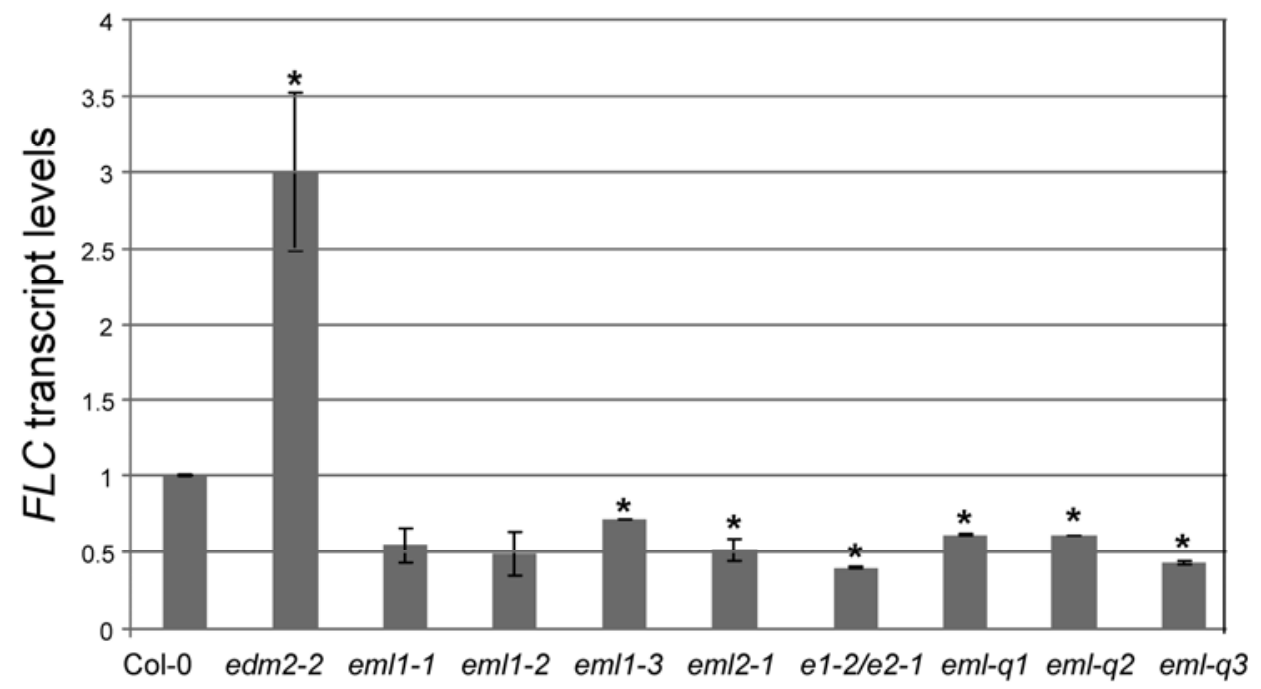

Fig. 4. FLC transcript levels in 2-week-old seedlings of the indicated Arabidopsis genotypes grown under long-day conditions determined by quantitative real-time reverse-transcription polymerase chain reaction. Error bars represent standard deviations. Data shown are based on averages from two biological replicates, each representing three technical replicates. Error bars represent standard deviations; * symbols mark significant changes $(P<0.05)$ relative to Col-0 as determined by Student's $t$ tests, which were performed as described previously (Yuan et al. 2006). 
transcription factor $\mathrm{R}$ in the control of anthocyanin accumulation (Hernandez et al. 2007). The ENT domain was originally identified in the human oncoprotein EMSY, which appears to counteract the transcriptional activator function of the tumor suppressor BRCA2 (Hughes-Davies et al. 2003). The observation that EMSY also interacted with proteins involved in chromatin remodeling such as HP1 and BS69 (Hughes-Davies et al. 2003), as well as the fact that BRCA2 has histone acetyltransferase activity (Raouf et al. 2005; Siddique et al. 1998), linked EMSY to the regulation of chromatin dynamics. In addition to the N-terminal ENT domain, AtEML1/2/3/4 harbor a central Agenet domain, which is related to epigenetic "reader" domains, such as the chromo domain or the tudor domain (Maurer-Stroh et al. 2003). Based on this, a general function of AtEML1/2/3/4 as epigenetic chromatin-associated factors is highly likely.

Consistent with the ability of AtEML1 and AtEML2 to physically interact in $N$. benthamiana nuclei with the race-specific defense regulator EDM2, we found these two AtEML members and possibly at least one additional representative of this family to contribute to the immune function of $E D M 2$. Functional links between EDM2 and AtEML1/2/3/4 members are further supported by the involvement of AtEML1 and $A t E M L 2$ in flowering time control, which is a second process to which EDM2 contributes. Whereas EDM2 and AtEML1/2/3/4 members promote resistance to $H$. arabidopsidis Hiks1 and $R P P 7$ expression, they have antagonistic functions in the control of floral transition and FLC expression. Our study did not address whether direct physical interactions between EDM2 and AtEML1/2/3/4 proteins are relevant for any of these functions (defense and flowering). However, a hypothetical scenario explaining how two physically interacting proteins can cooperate in one process and antagonize each other in another process may be that the existence of a complex of both partners is sufficient for cooperation in the first case (e.g., defense), whereas counteracting activities of both partners in the same or different complexes account for antagonism in the second case (e.g., flowering).

The role of AtEML1 and AtEML2 in floral transition resembles that of the protein kinase gene $W N K 8$, which also counteracts the promoting effect of EDM2 on this developmental step (Tsuchiya and Eulgem 2010a). In contrast to AtEML1 and AtEML2, $W N K 8$, however, appears not to affect the immune function of EDM2. Nevertheless, in Arabidopsis, a growing body of evidence hints at regulatory links between defense induction and floral transition. In addition to EDM2 and AtEML genes, several other defense components, including SA, have been found to affect flowering time control (Jin et al. 2008; March-Diaz et al. 2008; Tör et al. 2002; Wang et al. 2011). Therefore, functional links between these seemingly unrelated processes are unlikely to be coincidental. Pathogens with biotrophic or hemibiotrophic lifestyles are known to manipulate host development for their own benefit (Kay et al. 2007; Kazan and Manners 2009; Uchida and Tasaka 2010). In some cases, plants may take advantage of pathogen interference with host developmental programs and use such interference as a trigger for defense activation. Numerous studies have reported on regulatory circuits between pathogen-manipulated host processes and defense induction (Dodds and Rathjen 2010; Jones and Dangl 2006; Romer et al. 2007). Alternatively, plants may benefit from the modulation of their own developmental programs during pathogen infections and may "intentionally" accelerate or delay certain stages of their life cycle (Korves and Bergelson 2003). This may enable them to balance the allocation of energy and metabolic resources to immune responses with the need to properly maintain homeostasis of their own cells and tissues. It may even be an advantage for infected plants to speed up the transition to reproduction and seed production in order to ensure reproductive success during disease. Scenarios in which plants actively change their own development in response to pathogen infections are supported by observations that abiotic stresses can have similar effects (Balasubramanian et al. 2006; Franks 2011; Kolar and Senkova 2008). Focused studies on regulatory connections between plant immune responses and developmental programs may reveal many interesting surprises in the future.

Intriguingly, members of the AtEML1/2/3/4 gene set appear to have a wider role in immunity than $E D M 2$, whose defenserelated role seems to be limited to race-specific resistance against $H$. arabidopsidis Hiks1. Like the proteins encoded by AtEML1/2/3/4, primary structural features of EDM2, such as putative PHD-finger like epigenetic reader modules (Eulgem et al. 2007), point to a role of this protein in chromatin-associated transcriptional regulation. Thus, possible cooperation of AtEML1/2/3/4 and EDM2 at the level of chromatin dynamics may link race-specific pathogen recognition to general defense mechanisms. A broad role of AtEML1/2/3/4 in defense induction is consistent with the fact that such a function has also been demonstrated for BRCA2a, the Arabidopsis ortholog of the human EMSY-interactor BRCA2. BRCA2a was recently shown to be required for basal defense and to have a broad role in defense gene induction (Wang et al. 2010). It remains to be shown, however, to what extent details of the interactions between EMSY-like proteins and BRCA2 homologs and their regulatory roles are conserved between animal and plant systems.

Although the involvement of chromatin-associated regulation in flowering time control has been extensively examined (Dennis and Peacock 2007; He et al. 2003), the connection between these types of epigenetic processes and plant immunity is new and, thus far, only marginally explored. Only a small number of publications thus far have touched on this promising nascent area (Jaskiewicz et al. 2011; Palma et al. 2010; van den Burg and Takken 2009). With AtEML1/2/3/4, we have described here four interesting new components of the plant immune system that are likely to have roles in the regulation of chromatin states. In the case of floral transition, posttranslational modifications of nucleosome core histones provide an epigenetic memory of expanded vernalization, which conditions some Arabidopsis accessions to flower after subsequent exposure to long-day conditions. Similarly, chromatin modifications have recently been shown to prime some defense genes after an initial defense-related stimulus to respond more intensely to secondary defense induction (Jaskiewicz et al. 2011). Whether or not AtEML1/2/3/4 serve epigenetic memory processes in the control of flowering and defense induction will be an interesting subject of future studies.

\section{MATERIALS AND METHODS}

Yeast two-hybrid screens.

Yeast two-hybrid screens were performed as described previously (Tsuchiya and Eulgem 2010a).

\section{BiFC assays and subcellular localization of GFP-fusion proteins.}

BiFC assays were performed as described previously (Tsuchiya and Eulgem 2010a). Briefly, each cDNA of fulllength EDM2, AtEML1-v1, or AtEML-v3 was amplified by PCR and cloned into BiFC Gateway vectors (Citovsky et al. 2006) to examine in planta interactions between EDM 2 and AtEML-v1 or AtEML-v3. Expression cassettes to produce

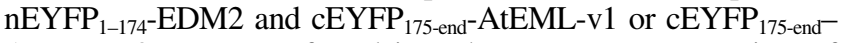
AtEML-v3 were transferred into the I-SceI or I-CeuI sites of 
the pPZP-RCS1 binary vector (Goderis et al. 2002). Agrobacterium tumefaciens GV3101 harboring the resulting BiFC binary vectors and a 19 silencing suppressor construct were coinfiltrated into the abaxial surface of 2- to 3-week-old $N$. benthamiana leaves as described previously (Goodin et al. 2002). YFP fluorescence was visualized in epidermal cell layers after 2 to 4 days of infiltration, using a Leica SP2 confocal microscope. Leaves were also infiltrated with 4',6-diamidino-2-phenylindole at $15 \mu \mathrm{g} / \mathrm{ml}$ for nuclei staining. Open reading frames of AtEML1-v1 or AtEML-v3 are also introduced into the pMDC43 vector (Curtis and Grossniklaus 2003) to produce GFP-AtEML1-v1 or GFP-AtEML1-v3. GFP florescence was observed as described above.

\section{Mutant lines.}

Wild-type plants of the Arabidopsis accession Col-0 were obtained from the Arabidopsis Biological Resource Center (ABRC; Ohio State University, Columbus, U.S.A.). All experiments were performed exclusively in the Col-0 background. We obtained all used T-DNA mutants (Table 1) from the SALK T-DNA insertion collection from the ABRC (Alonso et al. 2003). The edm2-2 allele has been described previously (Eulgem et al. 2007). The T-DNA insertion sites were confirmed by PCR-based genotyping using T-DNA border primers and gene-specific primers (Supplementary Table S1). Sequencing of T-DNA borders revealed errors in positions of T-DNA insertions at The Arabidopsis Information Resource database, such that the T-DNA insertion in emll-1 was found in the first intron while it was annotated in the second exon. The T-DNA insertion positions determined by sequencing are represented in Supplementary Figure S4. The eml1/eml2 double mutant was produced by crossing eml2-1 to emll-2 and genotyping their F2 progeny by PCR amplification.

\section{Transgenic lines.}

An amiRNA specifically targeting both AtEML3 and AtEML4 was designed using the Web MicroRNA Designer online tool against sequences conserved between these two genes (AT5G13020 and AT2G44440). To engineer the amiRNA, a total of six primers (A, B, I, II, III, and IV) were employed to initially perform three separate PCR assays using pRS300 (Schwab et al. 2006) as the template. A final overlapPCR reaction using the flanking primer pair (A and $\mathrm{B}$ ) was performed on the three primary PCR products as the template. To facilitate cloning into the Gateway system, we introduced $a t t \mathrm{~B} 1$ and $a t t \mathrm{~B} 2$ sites into primer $\mathrm{A}$ and $\mathrm{B}$, respectively. The final PCR product was cloned into pDONR/Zeo (Invitrogen, Carlsbad, CA, U.S.A.). After sequence confirmation, the insert was recombined into the Gateway destination vector pEarleyGate100 (Earley et al. 2006) through LR-clonase (Invitrogen). pEarleyGate-EML was mobilized into the A. tumefaciens GV3101 and was used to transform the eml1/eml2 double mutant by the floral dipping method (Clough and Bent 1998). The transformants were identified through BASTA selection. Transgenic $n a h G$ plants expressing an SA hydroxylase have been described previously (Delaney et al. 1994).

\section{RNA isolation and reverse-transcription PCR analysis.}

Total RNA was isolated from 2-week-old seedlings using Trizol reagent (Invitrogen). Total RNA $(1 \mu \mathrm{g})$ was incubated with 1 unit of DNase I, RNase-free (Fermentas, Glen Burnie, MD, U.S.A.) in a total volume of $10 \mu \mathrm{l}$ at $37^{\circ} \mathrm{C}$ for $30 \mathrm{~min}$. After inactivation of DNase I by adding $1 \mu \mathrm{l}$ of $50 \mathrm{mM}$ EDTA and heating to $65^{\circ} \mathrm{C}$ for $10 \mathrm{~min}$, RNA was reverse transcribed using 200 units of Maxima reverse transcriptase (Fermentas) and 100 pmol of oligo $(\mathrm{dT})_{18}$ primers in a sample volume of 20 $\mu l$. cDNA aliquots $(0.5 \mu \mathrm{l})$ were used as template for real-time
qPCR using an iQ5 real-time PCR detection system (Bio-Rad, Hercules, CA, U.S.A.). Each $25 \mu \mathrm{l}$ of reaction mix contained $12.5 \mu \mathrm{l}$ of Maxima SYBR green/fluorescein qPCR master mix (Fermentas) and $0.6 \mu \mathrm{M}$ gene-specific primers. Agarose gel electrophoresis and thermal denaturation (melt curve analysis) were used to confirm specific replicon formation. For each reaction, the threshold cycle value $(\mathrm{Ct})$ was determined by setting the threshold within the logarithmic amplification phase. Serial dilutions of total cDNA were used to calculate the amplification efficiency of each primer set according to the equation $E=10^{-1}$ /slope. Relative gene expression data were generated by the comparative $\mathrm{Ct}$ method using Actin 8 as a reference gene (Pfaffl 2001). All of the quantification experiments were done with at least two biological replicates in triplicate. All the primers were validated, and only those with efficiencies between 90 and $100 \%$ were used.

\section{H. arabidopsidis isolates and infections.}

The H. arabidopsidis isolates Hiks1 and Noco2 were described previously (Holub et al. 1994; Parker et al. 1993). $H$. arabidopsidis was grown, propagated, and applied to Arabidopsis as described previously (McDowell et al. 2000). Using Preval sprayers (Preval, Coal City, IL, U.S.A.), 1- or 3-weekold seedlings were spray inoculated with spore suspensions of H. arabidopsidis Hiks1 $\left(5 \times 10^{4}\right.$ spores $\left./ \mathrm{ml}\right)$ or $H$. arabidopsidis Noco2 $\left(2 \times 10^{4}\right.$ spores $\left./ \mathrm{ml}\right)$, respectively. Plants were scored at 7 days after infection for severity of infection by lactophenol trypan blue staining (Koch and Slusarenko 1990) or counting spores per plant fresh weight using a hemocytometer.

\section{Flowering time measurement}

Arabidopsis plants were grown at $22^{\circ} \mathrm{C}$ under long-day conditions ( $16 \mathrm{~h}$ of light and $8 \mathrm{~h}$ of darkness). Flowering time was measured as the number of rosette leaves formed at bolting.

\section{ACKNOWLEDGMENTS}

We thank Y.-H. Wei for critical reading of the manuscript; C. S. Pikaard (Washington University) for the pEarleyGate 100 vector; D. Weigel (Max Planck Institute) for the pRS300 plasmid; M. D. Curtis (University of Zürich) for the pMDC43 vector; S. B. Gelvin (Purdue University) for BiFC vectors; E. Holub (Warwick HRI, U.K.) and J. Parker (Max-PlanckInstitute, Cologne, Germany) for kindly providing the $H$. arabidopsidis isolates Hiks1 and Noco2, respectively; and ABRC stock center for providing Arabidopsis T-DNA mutants. This work was funded by National Science Foundation grant IOS-0724838-003 to T. Eulgem.

\section{LITERATURE CITED}

Alonso, J. M., Stepanova, A. N., Leisse, T. J., Kim, C. J., Chen, H., Shinn, P., Stevenson, D. K., Zimmerman, J., Barajas, P., Cheuk, R., Gadrinab, C., Heller, C., Jeske, A., Koesema, E., Meyers, C. C., Parker, H., Prednis, L., Ansari, Y., Choy, N., Deen, H., Geralt, M., Hazari, N., Hom, E., Karnes, M., Mulholland, C., Ndubaku, R., Schmidt, I., Guzman, P., Aguilar-Henonin, L., Schmid, M., Weigel, D., Carter, D. E., Marchand, T., Risseeuw, E., Brogden, D., Zeko, A., Crosby, W. L., Berry, C. C., and Ecker, J. R. 2003. Genome-wide insertional mutagenesis of Arabidopsis thaliana. Science 301:653-657.

Balasubramanian, S., Sureshkumar, S., Lempe, J., and Weigel, D. 2006. Potent induction of Arabidopsis thaliana flowering by elevated growth temperature. PLoS Genet. 2:e106. Published online.

Chisholm, S. T., Coaker, G., Day, B., and Staskawicz, B. J. 2006. Hostmicrobe interactions: Shaping the evolution of the plant immune response. Cell 124:803-814.

Citovsky, V., Lee, L. Y., Vyas, S., Glick, E., Chen, M. H., Vainstein, A., Gafni, Y., Gelvin, S. B., and Tzfira, T. 2006. Subcellular localization of interacting proteins by bimolecular fluorescence complementation in planta. J. Mol. Biol. 362:1120-1131.

Clough, S. J., and Bent, A. F. 1998. Floral dip: A simplified method for Agrobacterium-mediated transformation of Arabidopsis thaliana. Plant J. 16:735-743.

Curtis, M. D., and Grossniklaus, U. 2003. A gateway cloning vector set for 
high-throughput functional analysis of genes in planta. Plant Physiol. 133:462-469.

Delaney, T., Uknes, S., Vernooij, B., Friedrich, L., Weymann, K., Negrotto, D., Gaffney, T., Gut-Rella, M., Kessman, H., Ward, E., and Ryals, J. 1994. A central role of salicylic acid in plant disease resistance. Science 266:1247-1250.

Dennis, E. S., and Peacock, W. J. 2007. Epigenetic regulation of flowering. Curr. Opin. Plant Biol. 10:520-527.

Dodds, P. N., and Rathjen, J. P. 2010. Plant immunity: Towards an integrated view of plant-pathogen interactions. Nat. Rev. Genet. 11:539-548.

Earley, K. W., Haag, J. R. Pontes, O., Opper, K., Juehne, T., Song, K., and Pikaard, C. S. 2006. Gateway-compatible vectors for plant functional genomics and proteomics: pEarleyGates, pHellsgates and alternative destinations. Plant J. 45:616-629.

Eulgem, T., Weigman, V. J., Chang, H.-S., McDowell, J. M., Holub, E. B., Glazebrook, J., Zhu, T. A., and Dangl, J. L. 2004. Gene expression signatures from three genetically separable resistance gene signaling pathways for downy mildew resistance. Plant Physiol. 135:1129-1144.

Eulgem, T., Tsuchiya, T., Wang, X., Cuzick, A., Beasley, B., Toer, M., McDowell, J. M., Holub, E., Zhu, T., and Dangl, J. L. 2007. EDM2 is required for RPP7-dependent disease resistance in Arabidopsis and affects RPP7 transcript levels. Plant J. 49:829-839.

Franks, S. J. 2011. Plasticity and evolution in drought avoidance and escape in the annual plant Brassica rapa. New Phytol.

Glazebrook J. 2005. Contrasting mechanisms of defense against biotrophic and necrotrophic pathogens. Annu. Rev. Phytopathol. 43:205-227.

Goderis, I. J., De Bolle, M. F., Francois, I. E., Wouters, P. F., Broekaert, W. F., and Cammue, B. P. 2002. A set of modular plant transformation vectors allowing flexible insertion of up to six expression units. Plant Mol. Biol. 50:17-27.

Goodin, M. M., Dietzgen, R. G., Schichnes, D., Ruzin, S., and Jackson, A O. 2002. pGD vectors: Versatile tools for the expression of green and red fluorescent protein fusions in agroinfiltrated plant leaves. Plant $\mathbf{J}$. $31: 375-383$

Hartl, D., and Jones, E. W. 1998. Genetics-Principles and Analysis. Book, Jones and Bartlett Publishers, Sudbury, MA, U.S.A.

He, Y., Michaels, S. D., and Amasino, R. M. 2003. Regulation of flowering time by histone acetylation in Arabidopsis. Science 302:1751-1754.

Hernandez, J. M., Feller, A., Morohashi, K., Frame, K., and Grotewold, E. 2007. The basic helix loop helix domain of maize $\mathrm{R}$ links transcriptional regulation and histone modifications by recruitment of an EMSYrelated factor. Proc. Natl. Acad. Sci. U.S.A. 104:17222-17227.

Holub, E. B., and Beynon, J. L. 1996. Symbiology of mouse ear cress (Arabidopsis thaliana) and oomycetes. Adv. Bot. Res. 24:228-273.

Holub, E. B., Beynon, J. L., and Crute, I. R. 1994. Phenotypic and genotypic characterization of interactions between isolates of Peronospora parasitica and accessions of Arabidopsis thaliana. Mol. Plant-Microbe Interact. 7:223-239

Hughes-Davies, L., Huntsman, D., Ruas, M., Fuks, F., Bye, J., Chin, S. F., Milner, J., Brown, L. A., Hsu, F., Gilks, B., Nielsen, T., Schulzer, M., Chia, S., Ragaz, J., Cahn, A., Linger, L., Ozdag, H., Cattaneo, E., Jordanova, E. S., Schuuring, E., Yu, D. S., Venkitaraman, A., Ponder, B., Doherty, A., Aparicio, S., Bentley, D., Theillet, C., Ponting, C. P., Caldas, C., and Kouzarides, T. 2003. EMSY links the BRCA2 pathway to sporadic breast and ovarian cancer. Cell 115:523-535.

Jaskiewicz, M., Conrath, U., and Peterhansel, C. 2011. Chromatin modification acts as a memory for systemic acquired resistance in the plant stress response. EMBO (Eur. Mol. Biol. Organ.) Rep. 12:50-55.

Jin, J. B., Jin, Y. H., Lee, J., Miura, K., Yoo, C. Y., Kim, W. Y., Van Oosten, M., Hyun, Y., Somers, D. E., Lee, I., Yun, D. J., Bressan, R. A., and Hasegawa, P. M. 2008. The SUMO E3 ligase, AtSIZ1, regulates flowering by controlling a salicylic acid-mediated floral promotion pathway and through affects on FLC chromatin structure. Plant J. 53:530-540.

Jones, J. D., and Dangl, J. L. 2006. The plant immune system. Nature 444:323-329

Kay, S., Hahn, S., Marois, E., Hause, G., and Bonas, U. 2007. A bacterial effector acts as a plant transcription factor and induces a cell size regulator. Science 318:648-651.

Kazan, K., and Manners, J. M. 2009. Linking development to defense: Auxin in plant-pathogen interactions. Trends Plant Sci. 14:373-382.

Koch, E., and Slusarenko, A. J. 1990. Arabidopsis is susceptible to infection by a downy mildew fungus. Plant Cell 2:437-445.

Kolar, J., and Senkova, J. 2008. Reduction of mineral nutrient availability accelerates flowering of Arabidopsis thaliana. J. Plant Physiol. 165:1601-1609.

Korves, T. M., and Bergelson, J. 2003. A developmental response to pathogen infection in Arabidopsis. Plant Physiol. 133:339-347.
March-Diaz, R., Garcia-Dominguez, M., Lozano-Juste, J., Leon, J Florencio, F. J., and Reyes, J. C. 2008. Histone H2A.Z and homologues of components of the SWR1 complex are required to control immunity in Arabidopsis. Plant J. 53:475-487.

Maurer-Stroh, S., Dickens, N. J., Hughes-Davies, L., Kouzarides, T., Eisenhaber, F., and Ponting, C. P. 2003. The tudor domain 'Royal Family': Tudor, plant Agenet, chromo, PWWP and MBT domains. Trends Biochem. Sci. 28:69-74.

McDowell, J. M., Cuzick, A., Can, C., Beynon, J., Dangl, J. L., and Holub, E. B. 2000. Downy mildew (Peronospora parasitica) resistance genes in Arabidopsis vary in functional requirements for NDR1, EDS1, NPR1, and salicylic acid accumulation. Plant J. 22:523-530.

Nimchuk, Z., Eulgem, T., Holt, I. B., and Dangl, J. L. 2003. Recognition and response in the plant immune system. Annu. Rev. Genet. 37:579609.

Palma, K., Thorgrimsen, S., Malinovsky, F. G., Fiil, B. K., Nielsen, H. B., Brodersen, P., Hofius, D., Petersen, M., and Mundy, J. 2010. Autoimmunity in Arabidopsis acd11 is mediated by epigenetic regulation of an immune receptor. PLoS Pathog. 6:e1001137. Published online.

Parker, J. E., Szabò, V., Staskawicz, B. J., Lister, C., Dean, C., and Jones, J. D. G. 1993. Phenotypic characterization and molecular mapping of the Arabidopsis thaliana locus RPP5, determining resistance to Peronospora parasitica. Plant J. 4:821-833.

Pfaffl, M. W. 2001. A new mathematical model for relative quantification in real-time RT-PCR. Nucleic Acids Res. 29:e45.

Raouf, A., Brown, L., Vrcelj, N., To, K., Kwok, W., Huntsman, D., and Eaves, C. J. 2005. Genomic instability of human mammary epithelial cells overexpressing a truncated form of EMSY. J. Natl. Cancer Inst. 97:1302-1306.

Romer, P., Hahn, S., Jordan, T., Strauss, T., Bonas, U., and Lahaye, T. 2007. Plant pathogen recognition mediated by promoter activation of the pepper Bs3 resistance gene. Science 318:645-648.

Sato, M., Tsuda, K., Wang, L., Coller, J., Watanabe, Y., Glazebrook, J., and Katagiri, F. 2010. Network modeling reveals prevalent negative regulatory relationships between signaling sectors in Arabidopsis immune signaling. PLoS Pathog. 6:e1001011. Published online.

Schwab, R., Ossowski, S., Warthmann, N., and Weigel, D. 2006. Directed gene silencing with artificial microRNAs. Methods Mol. Biol. 592:71 88

Segonzac, C., and Zipfel, C. 2011. Activation of plant pattern-recognition receptors by bacteria. Curr. Opin. Microbiol. 14:54-61.

Siddique, H., Zou, J. P., Rao, V. N., and Reddy, E. S. 1998. The BRCA2 is a histone acetyltransferase. Oncogene 16:2283-2285.

Tör, M., Gordon, P., Cuzick, A., Eulgem, T., Sinapidou, E., Mert, F., Can, C., Dangl, J. L., and Holub, E. B. 2002. Arabidopsis SGT1b is required for defense signaling conferred by several Downy Mildew (Peronospora parasitica) resistance genes. Plant Cell 14:993-1003.

Torres, M. A., Dang1, J. L., and Jones, J. D. G. 2002. Arabidopsis gp91phox homologues AtrbohD and AtrbohF are required for accumulation of reactive oxygen intermediates in the plant defense response. Proc. Natl. Acad. Sci. U.S.A. 99:523-528.

Tsuchiya, T., and Eulgem, T. 2010a. The Arabidopsis defense component EDM2 affects the floral transition in an FLC-dependent manner. Plant J. 62:518-528.

Tsuchiya, T., and Eulgem, T. 2010b. Co-option of EDM2 to distinct regulatory modules in Arabidopsis thaliana development. BMC Plant Biol. $10: 203$

Tsuda, K., Sato, M., Stoddard, T., Glazebrook, J., and Katagiri, F. 2009. Network properties of robust immunity in plants. PLoS Genet. 5:e1000772. Published online.

Uchida, N., and Tasaka, M. 2010. Intersections between immune responses and morphological regulation in plants. J. Exp. Bot. 61:2539-2547.

van den Burg, H. A., and Takken, F. L. 2009. Does chromatin remodeling mark systemic acquired resistance? Trends Plant Sci. 14:286-294.

Wang, G. F., Seabolt, S., Hamdoun, S., Ng, G., Park, J., and Lu, H. 2011. Multiple roles of WIN3 in regulating disease resistance, cell death, and flowering time in Arabidopsis. Plant Physiol. 156:1508-1519

Yuan, J. S., Reed, A., Chen, F., and Stewart, C. N. 2006. Statistical analysis of real-time PCR data. BMC Bioinf. 7:85.

\section{AUTHOR-RECOMMENDED INTERNET RESOURCES}

The Bio-Array Resource server: bar.utoronto.ca/welcome.htm

The Arabidopsis Information Resource (TAIR) database: www.arabidopsis.org

Web MicroRNA Designer online tool: wmd.weigelworld.org 